\title{
Practical Implementation of Spatial Modulation
}

\author{
Nikola Serafimovski, Abdelhamid Younis, Raed Mesleh, Senior Member, IEEE, P. Chambers, \\ Marco Di Renzo, Member, IEEE, Cheng-Xiang Wang, Senior Member, IEEE, \\ Peter M. Grant, Fellow, IEEE, Mark A. Beach, and Harald Haas, Member, IEEE
}

\begin{abstract}
In this paper, we seek to characterize the performance of spatial modulation (SM) and spatial multiplexing (SMX) with an experimental testbed. Two National Instruments (NI) PXIe devices are used for the system testing: one for the transmitter and one for the receiver. The digital signal processing (DSP) that formats the information data in preparation for transmission is described, along with the DSP that recovers the information data. In addition, the hardware limitations of the system are also analyzed. The average bit-error ratio (ABER) of the system is validated through both theoretical analysis and simulation results for SM and SMX under the line-of-sight (LoS) channel conditions.
\end{abstract}

Index Terms-Experimental results, multiple-input multipleoutput (MIMO) systems, spatial modulation (SM), spatial multiplexing (SMX), wireless testbed.

\section{INTRODUCTION}

$\mathbf{M}$ ULTIPLE-INPUT multiple-output (MIMO) systems offer a significant increase in spectral efficiency in comparison with single-antenna systems [1], [2]. An example is spatial modulation (SM), which increases the spectral efficiency of single-antenna systems while avoiding interchannel interference (ICI) [3]. This is attained, as shown in Fig. 1, through the adoption of a new modulation and coding scheme, which foresees the activation, at each time instance, of a single antenna that transmits a given data symbol (constellation symbol) and the exploitation of the spatial position (index) of the active antenna as an additional dimension for data

Manuscript received October 2, 2012; revised February 18, 2013 and April 24, 2013; accepted May 2, 2013. Date of publication June 6, 2013; date of current version November 6, 2013. This work was supported in part by The University of Edinburgh Initiating Knowledge Transfer Fund, the Engineering and Physical Sciences Research Council Fellowship under Grant EP/K008757/1, by the Research Councils U.K. under Grant EP/G042713/1 [UK-China Science Bridges "R\&D on (B)4G Wireless Mobile Communications"], by the European Union under Grant PITNGA2010264759 (GREENET), and by the Key Laboratory of Cognitive Radio and Information Processing, Guilin University of Electronic Technology, Ministry of Education, China, under Grant 2013KF01. The review of this paper was coordinated by Prof. Y. L. Guan.

N. Serafimovski, A. Younis, P. M. Grant, and H. Haas are with The University of Edinburgh, Edinburgh EH9 3JL, U.K. (e-mail: n.serafimovski@ed.ac.uk; a.younis@ed.ac.uk; p.grant@ed.ac.uk; h.haas@ed.ac.uk).

R. Mesleh is with the Department of Electrical Engineering and the Sensor Networks and Cellular Systems Research Center, University of Tabuk, Tabuk, Saudi Arabia (e-mail: rmesleh.sncs@ut.edu.sa).

P. Chambers and C.-X. Wang are with Heriot-Watt University, Edinburgh EH14 4AS, U.K. (e-mail: P.Chambers@hw.ac.uk; Cheng-Xiang.Wang@hw. ac.uk).

M. Di Renzo is with the Laboratoire des Signaux et Systèmes, Unitè Mixte de Recherche 8506, Centre National de la Recherche Scientifique-École Supèrieure d'Électricitè-Universitè Paris-Sud XI, 91192 Gif-sur-Yvette, France (e-mail: marco.direnzo@1ss.supelec.fr).

M. A. Beach is with the University of Bristol, Bristol BS8 1UB, U.K. (e-mail: M.A.Beach@bristol.ac.uk).

Color versions of one or more of the figures in this paper are available online at http://ieeexplore.ieee.org.

Digital Object Identifier 10.1109/TVT.2013.2266619

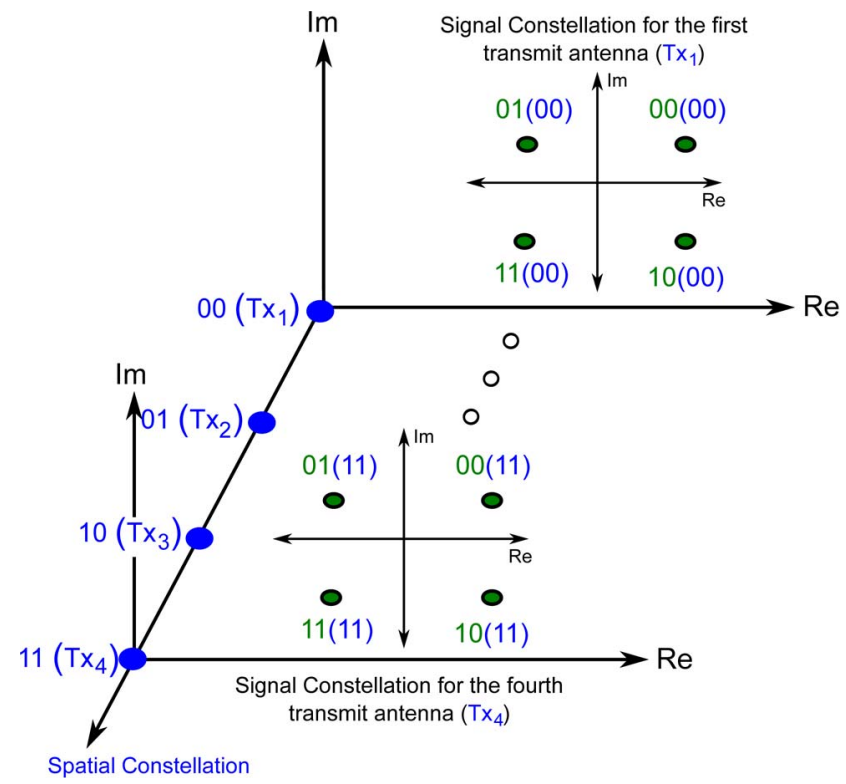

Fig. 1. Unique 3-D constellation diagram for SM. The lower two bits, in the 4-bit word, define the spatial-constellation point, which identifies the active antenna. These are shown in parentheses. The remaining two bits determine the signal-constellation point that is to be transmitted.

transmission (spatial symbol) [4]. Both the constellation and spatial symbols depend on the incoming data bits. An overall increase by the base-two logarithm of the number of transmit antennas of the spectral efficiency is achieved. This limits the number of transmit antennas to be a power of two, unless fractional-bit-encoded SM [5] or generalized SM (GSM) [6] is used. In particular, in [6], it is shown that the number of spatial symbols does not need to be equal to the number of transmit antennas. For example, if GSM is used, the number of spatial symbols is equal to the number of unique channel signatures between the transmitter and the receiver, where the unique channel signatures can be obtained by activating various combinations of the available transmit antennas. In this paper, however, these unique channel signatures are assumed to be due to the activation of individual transmit antennas.

Activating only one antenna at a time means that only one radio-frequency $(\mathrm{RF})$ chain is needed, which significantly reduces the hardware complexity of the system [7]. Moreover, the most energy-consuming parts of a base station (BS) are the power amplifiers and the RF chains that are associated with each transmitter [8], where the power requirements of a BS are shown to linearly increase with the number of RF chains that are added [9]. However, as only one RF chain is needed, SM offers a reduction in the energy consumption, which linearly scales with the number of transmit antennas [10], [11]. Furthermore, the computational complexity of the ML detector for SM 


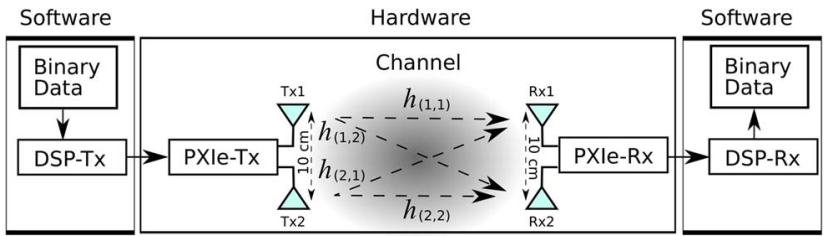

Fig. 2. Block sequence of the main steps in the experiment from the generation of the binary data to its recovery.

[SM-maximum likelihood (ML)] is equal to the complexity of single-input multiple-output (SIMO) systems [12], i.e., the complexity of SM-ML depends only on the spectral efficiency and the number of receive antennas and does not depend on the number of transmit antennas. Moreover, in [13]-[15], the complexity of SM is further reduced by using the sphere decoder (SD).

Several papers that seek to understand and improve the performance of SM in various scenarios are available in literature. In [16] and [17], the average bit-error ratio (ABER) performance of SM is improved by introducing trellis coding on the transmitting antennas. The optimal detector is derived with and without channel state information at the receiver in [12], [18], and [19]. The ABER performance is given when considering channel estimation errors in [20]-[22]. The optimal power allocation for the case of two transmit antennas and one receive antenna system is given in closed form in [23], and the ABER performance of SM in correlated fading channels is considered in [24]. In [25] and [26], spectral efficiency and diversity gains are obtained by combining SM with space-time block codes (STBC-SM). Applying SM to relaying systems is also shown to result in significant signal-to-noise-ratio (SNR) gains when compared with noncooperative decode and forward techniques [27]. In [28], the overall power performance of a BS employing SM is studied. More recently, a comprehensive analytical framework to compute the ABER of SM over generalized fading channels has been introduced in [29]. Moreover, in [30], for the first time, the performance of SM is analyzed using realworld channel measurements. The latest research achievements and an outline of some relevant open research issues for SM are reviewed in [31]. All research thus far is strictly theoretical.

In this paper, the ABER performance of SM is analyzed in a practical testbed and compared with that for spatial multiplexing (SMX). In particular, the National Instruments (NI) PXIe-1075 chassis are used at the transmitter and the receiver. The design of the testbed hardware and the software used are explained in detail, along with the transmission chain. The effects of the entire transmission chain on the system performance are examined. The basic elements of the transmission link are the transmit RF chain, the wireless channel, and the receive RF chain. In addition to the effects of the wireless channel on the phase and the amplitude of the signal, the impact on the system performance of the power imbalances (PIs) in the transmitter and receiver RF chains is discussed. Furthermore, an analytical upper bound for the ABER performance of SM over non-line-of-sight (NLoS) channels with PI is derived and compared with the experimental and computer simulation results. The experimental results validate the analytical bound, as well as the attained computer simulations. Finally, the performance

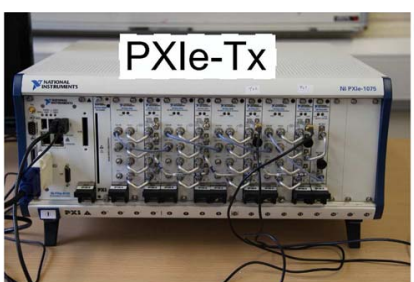

(a)

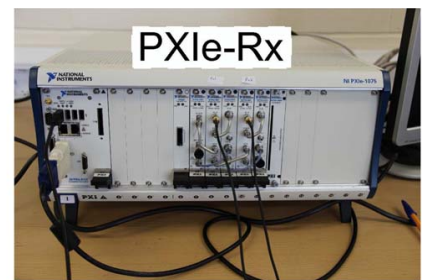

(b)
Fig. 3. NI-PXIe-1075 chassis with the relevant onboard modules at (a) the transmitter (PXIe-Tx) and (b) the receiver (PXIe-Rx).

of SM is compared with the theoretical and experimental results of SMX.

This paper is organized as follows: The system setup, equipment, and digital signal processing (DSP) are presented in Section II. The equipment constraints are then considered in Section III, while the analytical modeling is discussed in Section IV. In addition, the computational complexity of the SM decoder algorithm is presented in Section V. The performance of SM is then characterized in the experimental and simulation environments in Section VI, where it is compared with the theoretical and experimental results of an SMX system. Finally, this paper is summarized in Section VII.

\section{Testbed Setup and System Model}

The testbed setup and the transmission chain can be separated into software and hardware parts, as shown in Fig. 2. The hardware consists of the NI-PXIe chassis at the transmitter (PXIe-Tx) and the NI-PXIe chassis at the receiver (PXIe-Rx). The software consists of the DSP at the transmitter (DSP-Tx) and the DSP at the receiver (DSP-Rx).

The binary data to be broadcasted are first processed by the DSP-Tx, before being transmitted through the fading channel by the PXIe-Tx. The channel coefficient on the link between transmit antenna $n_{t}$ and receive antenna $r$ is denoted by $h_{\left(r, n_{t}\right)}$. Note that the number of antennas at the transmitter and the receiver are denoted by $N_{t}$ and $N_{r}$, respectively. At the receiver, the PXIe-Rx records the RF signal and passes it through to the DSP-Rx for processing, where the original data stream is recovered.

\section{A. Testbed Hardware}

The NI-PXIe-1075 chassis are equipped with a $1.8-\mathrm{GHz}$ Intel-i7 processor with 4-GB random access memory and are shown in Fig. 3. The system has two transmit antennas and two receive antennas. Each antenna at the transmitter and the receiver contains two quarter-wave dipoles and one half-wave dipole placed in the middle. All three dipoles are vertically polarized. In addition, each antenna has a peak gain of $7 \mathrm{dBi}$ in the azimuth plane, with an omnidirectional radiation pattern.

1) Transmitter Hardware (PXIe-Tx): The following NI-PXIe modules are used at the transmitter:

I) NI-PXIe-5450 16-bit I/Q Signal Generator (SG-16bit);

II) NI-PXIe-5652 RF Signal Generator with a $500-\mathrm{kHz}$ to 6.6-GHz frequency range (SG-RF);

III) NI-PXIe-5611 intermediate frequency to carrier RF upconverter (upconverter). 
The PXIe-Tx has an operational frequency range of $85 \mathrm{MHz}$ to $6.6 \mathrm{GHz}$ and can facilitate a bandwidth of $100 \mathrm{MHz}$ at a maximum transmission power of $5 \mathrm{dBm}$.

At the transmitter, the SG-16bit performs a linear mapping of the signed 16-bit range to the output power and polarization, i.e., the peak voltage amplitude is assigned to any value in the transmission vector that is equal to $2^{15}$ with a linear scale of the voltage amplitude down to zero. The output from SG-16bit then goes to SG-RF, which is connected to the upconverter. The upconverter outputs the analog waveform corresponding to the data resulting from DSP-Tx at a carrier frequency of $2.3 \mathrm{GHz}$. This completes a single RF chain. The transmission of the RF signal by the upconverters is synchronized by using a $10-\mathrm{MHz}$ reference signal.

2) Receiver Hardware (PXIe-Rx): The following NI-PXIe modules are used at the receiver:

I) NI-PXIe-5652 an onboard reference clock (SG-RF);

II) NI-PXIe-5622 16-bit digitizer, which records data samples in an I16 format (16-Bit Digitizer);

III) NI-PXIe-5601 RF downconverter (downconverter).

The PXIe-Rx can operate in a frequency range of $10 \mathrm{MHz}$ to $6.6 \mathrm{GHz}$ and can facilitate an operational bandwidth of $50 \mathrm{MHz}$. For more details about the specifications of each module, see [32] and [33].

At the receiver, each antenna is associated with a complete RF chain. For each antenna, the downconverter is used to detect the analog RF signal from its dedicated antenna. The signal is then sent to the dedicated 16-Bit Digitizer. The 16-Bit Digitizer applies a bandpass filter with a real flat bandwidth that is equal to $B_{f}=\left(0.4 \times f_{s}\right)$, where $f_{s}$ is the sampling rate [32]. The sampling rate in the experiment is $10 \mathrm{Ms} / \mathrm{s}$, which results in a real flat bandwidth of $4 \mathrm{MHz}$. This may result in frequency-selective fading. Nonetheless, equalization is not required for the detection of SM or SMX signals in this experiment because of the following: 1) There are no multitap delays in the experimental setup due to very small distance between the transmit and receiver antennas. 2) ML detection is used to decode the received signal for both SM and SMX. The use of ML detection is applied to the complete SM symbol, i.e., the spatial and signal symbols are jointly decoded. Finally, after the synchronization of the 16-Bit Digitizer with the onboard reference clock of the SG-RF, the 16-Bit Digitizer writes the received binary files. The simultaneous recording of the two signals coming from Tx1 and Tx2 is facilitated by utilizing multiple processing cores and multiple NI-PXIe modules. The recorded files are then processed according to DSP-Rx in Fig. 4.

\section{B. Testbed Software}

MATLAB was used to facilitate the DSP-Tx and the DSP-Rx. The DSP-Tx processes the information data and generates binary files that can be transmitted by the PXIe-Rx. The DSP-Rx processes the received data from the PXIe-Rx and recover the original information data stream. Fig. 4 outlines the processing algorithms at the DSP-Tx and the DSP-Rx.

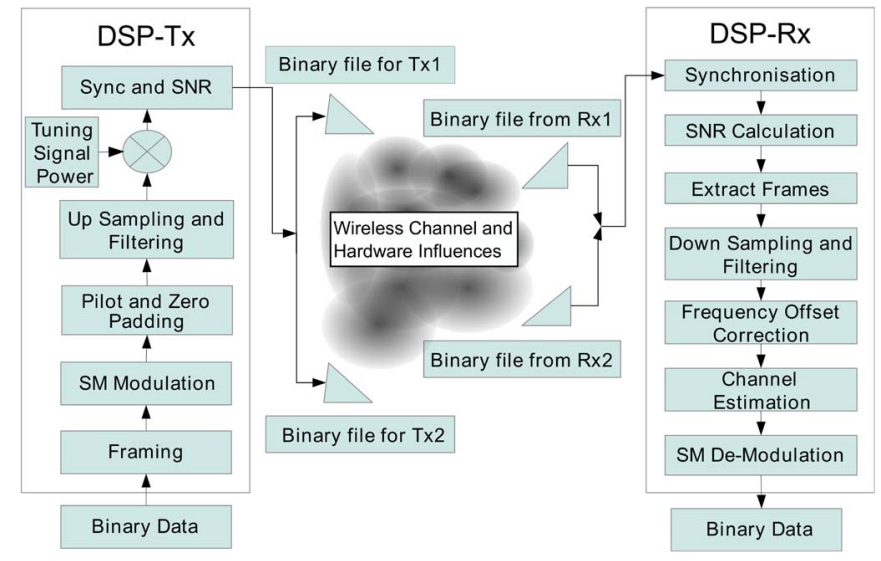

Fig. 4. Step-by-step layout of the binary data encoder (DSP-Tx) and decoder (DSP-Rx) processes.

1) DSP-Tx: The DSP-Tx process takes the incoming binary information data and performs the following.

1.1 Framing: The incoming data is split into frames consisting of 100 symbols per frame.

1.2 Modulation: The data in each frame is modulated using SM or SMX:

- SM: The bit stream is divided into blocks containing $\log _{2}\left(N_{t} M\right)$ bits each, where $M$ is the signalconstellation size. The following mapping rule is then used [4]:

a) The first $\log _{2}\left(N_{t}\right)$ bits determine which transmit antenna is active, i.e., they determine the spatial-constellation point of SM. In this paper, the transmit antenna broadcasting is denoted by $n_{t}$ with $n_{t} \in\left\{1,2, \ldots, N_{t}\right\}$.

b) The second $\log _{2}(M)$ bits are used to choose a symbol in the signal-constellation diagram. Without loss of generality, quadrature amplitude modulation (QAM) is considered. The actual complex symbol emitted by the transmit antenna $n_{t}$ is denoted by $s_{t}$, with $s_{t} \in\left\{s_{1}, s_{2}, \ldots, s_{M}\right\}$.

By following the aforementioned steps, the $N_{t} \times 1$ dimensional transmit vector is

$$
\mathbf{x}_{n_{t}}, s_{t}=\left[\mathbf{0}_{1 \times\left(n_{t}-1\right)}, s_{t}, \mathbf{0}_{1 \times\left(N_{t}-n_{t}\right)}\right]^{T}
$$

where $[\cdot]^{T}$ denotes the transpose operation, and $\mathbf{0}_{p \times q}$ is a $p \times q$ matrix with all-zero entries. Equation (1) is a representation of the transmission vector for SM. Since SM activates only one transmit antenna at any transmission instance, only one transmit antenna can broadcast a symbol, while all others remain silent. To this extent, the transmit vector is composed of all zeros, except for the single symbol $s_{t}$, which is broadcasted from antenna $n_{t}$. In this manner, SM avoids ICI and allows single-stream ML decoding. In addition, SM is energy efficient since only a single $\mathrm{RF}$ chain is active while still providing a multiplexing gain [10]. 
- SMX: In this case, the bit stream is divided into blocks of $N_{t} \log _{2}(M)$ bits; then, according to [34], the following mapping rule is used.

a) Each $\log _{2}(M)$ bit is separately modulated using $M$-QAM modulation.

b) The modulated symbols are then simultaneously transmitted from the $N_{t}$ transmit antennas.

1.3 Pilot and zero padding: The least-square (LS) channel estimation algorithm with local orthogonal pilot sequences is used to estimate the channel [35]. Two pilot signals are added for each frame, one of which at the start of the frame and one at the end. Each pilot signal contains ten pilot sequences, where the orthogonal pilot sequence for the $n_{t}$ th transmit antenna is defined as

$$
\Theta_{n_{t}}(\ell)=\exp \left(2 \pi j \frac{n_{t} \ell}{N_{\Theta}}\right)
$$

where $\Theta_{n_{t}}(\ell)$ is the $\ell$ th element of the pilot sequence $\Theta_{n_{t}}$ transmitted from antenna $n_{t}, j=\sqrt{-1}$ is the imaginary unit, and $N_{\Theta}$ is the cardinality of the pilot sequence. In this paper, the length of each pilot sequence is $N_{\Theta}=10$. To avoid interframe interference, an all-zero sequence of 50 zero-valued symbols is added to both the start and the end of the frame. Furthermore, a sequence of constantvalued symbols is added to enable frequency-offset (FO) estimation at the receiver. The length of the FO estimation sequence is 1000 symbols.

1.4 Upsampling and filtering: Upsampling and matched filtering (pulse shaping) are used to maximize the SNR and reduce intersymbol interference [36]. Each frame is upsampled with an upsampling ratio of 4 and then passed through a root-raised-cosine (RRC) finite-impulseresponse (FIR) filter with 40 taps and a rolloff factor of 0.75 . The large rolloff factor is necessary to ensure that the power is focused in a short-time instance to ensure that only a single RF chain is active when using SM.

1.5 Tuning signal power: The SNR is varied by changing the power of the transmitted signal to obtain the ABER. This is done by multiplying each transmission vector with a "tuning signal power" factor to obtain the desired transmit power. In particular, the amplitude of the "data section" in the transmission vector is changed by using the "tuning signal power" factor.

1.6 Synchronization and SNR: Several preambleautocorrelation-based methods for frame synchronization were tested [37]-[39]. However, despite the introduction of an interpolation filter at the receiver and due to the channel attenuations, the estimated start of the signal was typically in error by one or two samples. This meant that sample synchronization could not be consistently achieved, resulting in off-by-one errors. The investigation of synchronization techniques is outside the scope of this paper, but to avoid synchronization via a cable, as is often done in similar experimental systems, the peak-detection technique has been applied, which resulted in the desired outcome. We recognize that this technique is suboptimal as it results in power-amplifier saturation and potential signal distortions. Nonetheless, a sequence of 20 symbols with maximum power, each of which is separated by 50 zero-valued symbols, are added to the start of the transmitted signal. The large power difference between the maximum power peaks and the power of the "data section" symbols is reasonable since the instantaneous channel power may fluctuate by as much as $20 \mathrm{~dB}$ due to fast fading [40], [41]. The power difference between the synchronization section and the remaining sections is set to be larger than the maximum channel variation. In this manner, successful peak detection is guaranteed. If this is not the case, no peak may be detected at the receiver, and all further decoding would be erroneous. To facilitate SNR calculations at the receiver, two sequences of power and no power are added after the synchronization pulses of the transmitted signal, which are indicated by the "SNR section" in Fig. 5. Each sequence contains five blocks of 50000 symbols and 50000 zeros. The first sequence is transmitted from the first antenna, whereas the second antenna is off. The second sequence is transmitted from the second antenna, whereas the first antenna is off.

After the DSP-Tx process is completed, the transmit vector symbols are converted to I16 format and are recorded to a binary file. This binary file is then broadcast by the PXIe-Tx.

Fig. 5 is an absolute value representation of the processed incoming data that are passed to the first transmit antenna (Tx1), and Fig. 6 shows the absolute value representation of each frame. Note that the "data section" is a series of concatenated frames. In Fig. 6, it is shown that each frame contains 26100 samples. Therefore, the period of each frame is $T_{\text {Frame }}=$ $26100 / f_{s}=2.6 \mathrm{~ms}$. This is much less than the coherence time of the channel given that, typically, the coherence time for a stationary indoor environment is approximately $7 \mathrm{~ms}$ (see [41], and references therein). Hence, the channel estimation at the receiver is valid for the frame duration.

2) DSP-Rx: The data that are received by the PXIe-Rx are processed by the DSP-Rx to recover the original data stream. To accomplish this, the following steps are required.

2.1 Synchronization: This is achieved by searching for the peaks with a value above a certain threshold in the received signal. The threshold is set as $70 \%$ of the highest value in the received vector. This threshold level accounts for the natural voltage variations in the system, i.e., the difference between the peak voltage and the root-mean-square voltage. If the number of peaks found is less than 20 , then the received vector is discarded from further calculations.

2.2 SNR calculation: The SNR is defined as

$$
\mathrm{SNR}=\frac{E\left[\|\mathbf{H} \mathbf{x}\|_{F}^{2}\right]}{\sigma_{n}^{2}}
$$

where $\mathbf{H}$ is the $N_{r} \times N_{t}$ channel matrix, $\mathbf{x}$ is the $N_{t} \times$ 1 transmitted vector, $E[\cdot]$ is the expectation operator, and $\|\cdot\|_{F}$ is the Forbenius norm. Assuming that the noise at the receiver is additive white Gaussian noise (AWGN), the received signal for the duration of the SNR sequence can be written as follows:

$$
\mathbf{y}=\mathbf{h}_{n_{t}} s_{t}+\mathbf{n}
$$




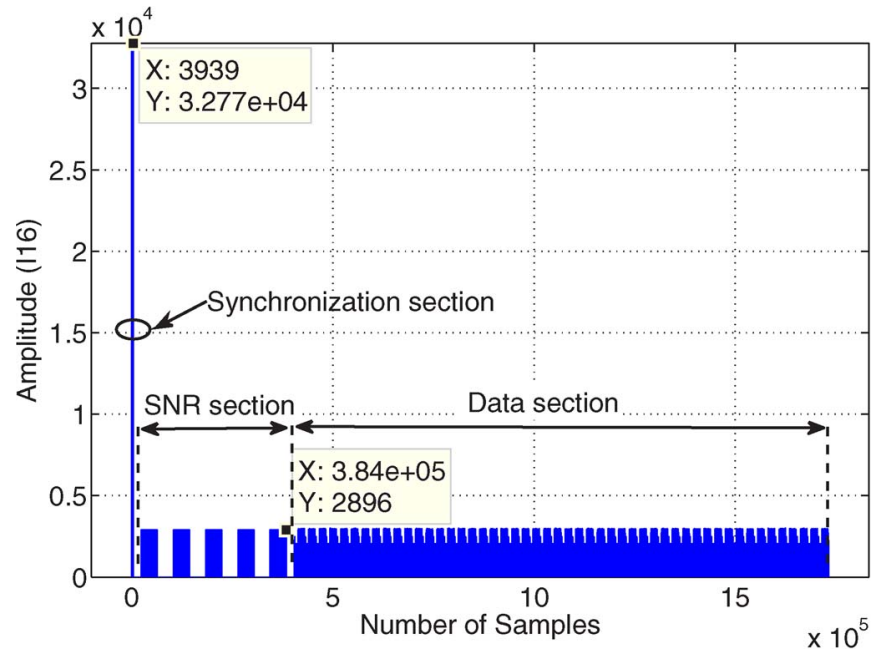

Fig. 5. Absolute value representation of the transmission vector being sent to Tx1. The synchronization, SNR estimation, and data sections are shown. The value of the peak must be equal to $2^{15}$ since the 16bit-Digitizer operates using an I16 format before tuning the signal power of the data. The highest value in the SNR section is the same as the highest value in the information data section, which in this example, is a value of 2896 . The peak value is $2^{15}$. There is approximately a $21.1 \mathrm{~dB}$ difference between the peak power in the synchronization section and the peak power in the SNR estimation and data sections. This is apparent when looking at the two data points shown in the figure.

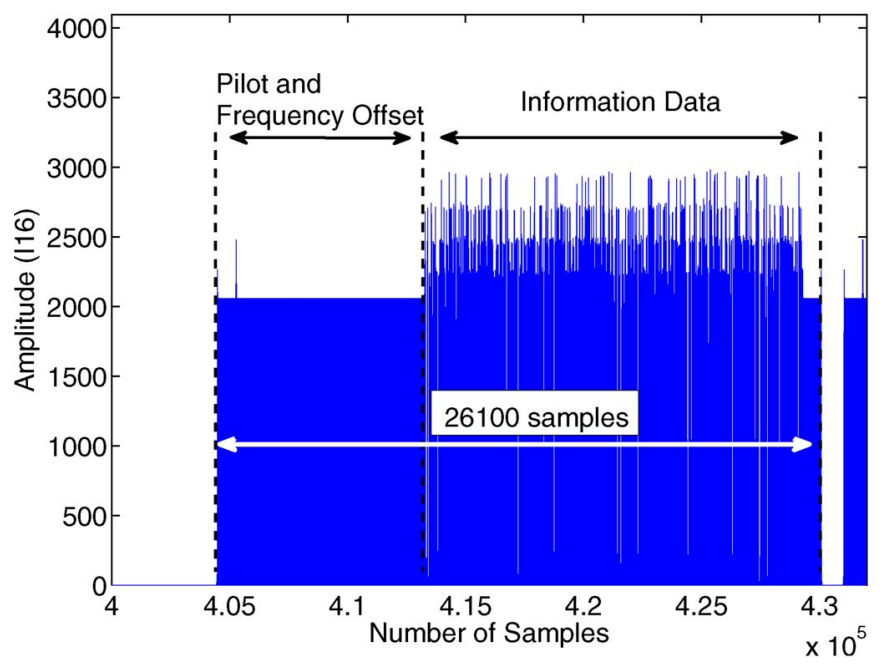

Fig. 6. Absolute value representation of a single frame from the vector being transmitted by Tx1 in the I16 data format, which is a signed 15-bit representation of an integer number.

where $\mathbf{y}$ is the $N_{r} \times 1$ received vector, $\mathbf{h}_{n_{t}}$ is the $n_{t}$ column of the channel matrix $\mathbf{H}, \mathbf{n}$ is the $N_{r} \times 1$ AWGN vector with $\sigma_{n}^{2}$ variance and $\mu_{n}$ mean, and $s_{t}$ is the transmitted symbol from the $n_{t}$ antenna. As mentioned in Section II-B1, only a single transmit antenna is active when broadcasting the SNR sequence, and $s_{t}$ is either equal to the maximum value in the "data section" $x_{\max }$ or zero, as shown in Fig. 5. Hence, the received signal in (4) can be rewritten as

$$
\mathbf{y}= \begin{cases}\mathbf{h}_{n_{t}} x_{\max }+\mathbf{n}, & s_{t}=x_{\max } \\ \mathbf{n}, & s_{t}=0 .\end{cases}
$$

Proceeding from (5)

$$
\begin{aligned}
E\left[\|\mathbf{H} \mathbf{x}\|_{F}^{2}\right] & =E\left[\|\mathbf{y}-\mathbf{n}\|_{F}^{2}\right] \\
\sigma_{n}^{2} & =E\left[\|\mathbf{n}\|_{F}^{2}\right]-E[\|\mathbf{n}\|]^{2}
\end{aligned}
$$

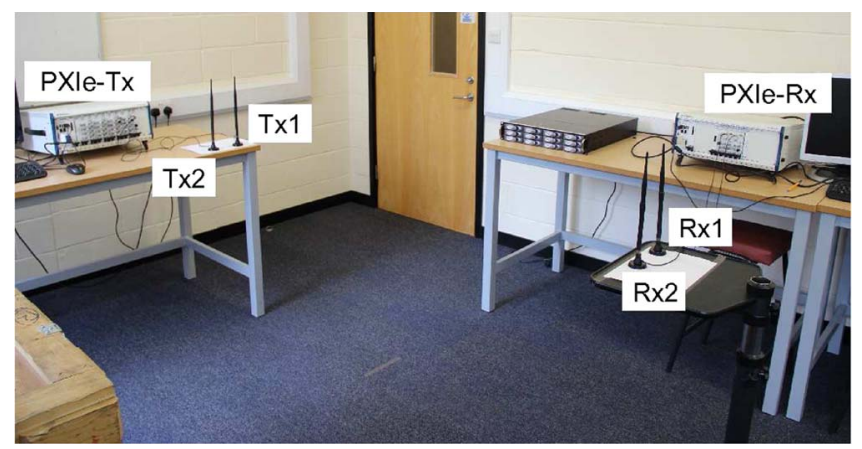

Fig. 7. Experimental setup in the laboratory.

where $[\cdot]^{H}$ is the Hermitian operation. As discussed in Section II-B1, each SNR sequence contains 50000 symbols and 50000 zero-valued symbols. Since the noise in the system represents an ergodic process, the ensemble average in (6) can be replaced with a time average, i.e.,

$$
\begin{aligned}
E\left[\|\mathbf{H} \mathbf{x}\|_{F}^{2}\right] & =\sum_{i=1}^{50000}\left(\left\|\mathbf{y}_{i}\right\|_{F}^{2}-\left\|\mathbf{n}_{i}\right\|_{F}^{2}-2 \mathbf{y}_{i}^{H} \mathbf{n}_{i}\right) \\
\sigma_{n}^{2} & =\sum_{i=1}^{50000}\left\|\mathbf{n}_{i}\right\|_{F}^{2}-\left[\sum_{i=1}^{50000}\left\|\mathbf{n}_{i}\right\|_{F}\right]^{2}
\end{aligned}
$$

where $\mathbf{y}_{i}$ and $\mathbf{n}_{i}$ are the $i$ th received vector. To get a more accurate estimation, the SNR is calculated for the five transmitted SNR sequences that are received at both antennas and then averaged again over those measurements.

2.3 Extract frames: After finding the start of the transmission and calculating the SNR, the DSP-Rx performs a serial-toparallel conversion to separate the received frames.

2.4 Downsampling and filtering: To complete the matched filter that is described in Section II-B1, each frame is downsampled by a factor of 4 and passed through an RRCFIR filter.

2.5 FO correction: The DSP-Rx estimates the FO for each frame by

$$
\Delta_{f}=\frac{\angle x_{1000}-\angle x_{1}}{2 \pi \times 1000}
$$

where $\angle x_{1000}, \angle x_{1}$ are the angles of the first and last samples of the FO sequence transmitted by the DSP-Tx, where the FO sequence has exactly 1000 symbols. These angle values are obtained by correcting the radian phase angles in a vector by adding multiples of $\pm 2 \pi$ as required. This enables a better estimate of the phase offset. Assuming a linear phase rotation, the FO can be estimated using (10). The FO for each frame is then corrected by

$$
\widetilde{y}_{i}=y_{i} \times e^{-j 2 \pi \Delta_{f} i}
$$

where $\widetilde{y}_{i}$ and $y_{i}$ are the $i$ th elements of the corrected and uncorrected received frames, respectively.

2.6 Channel estimation: The channel estimation is done by using the LS channel estimation algorithm that is proposed in [35], where, for each frame, the channel is estimated by

$$
\widetilde{\mathbf{H}}_{\mathrm{LS}}=\frac{1}{N_{\Theta}} \Theta^{H} \mathbf{H}_{r}
$$




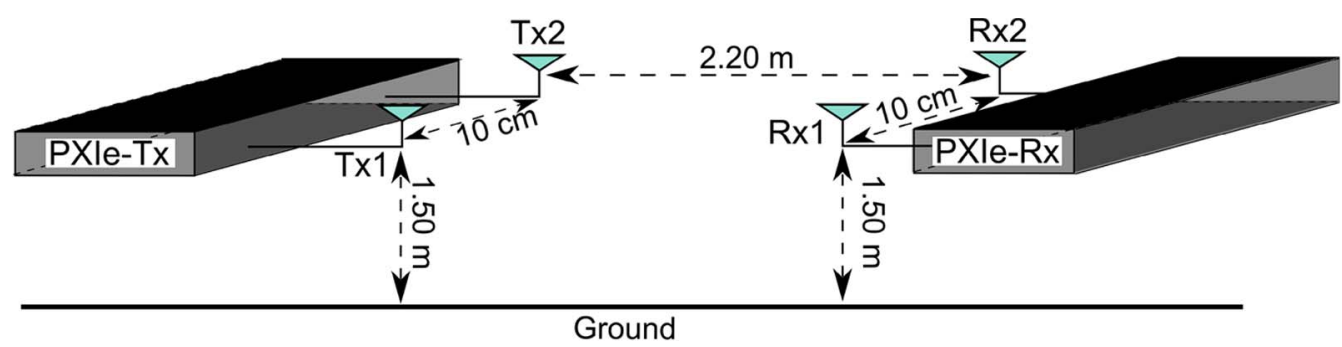

Fig. 8. Physical experimental layout. A pair of receive antennas and a pair of transmit antennas are set $2.2 \mathrm{~m}$ apart from each other with a direct LoS. Each pair of antennas is $1.5 \mathrm{~m}$ from the ground, and there is a $10-\mathrm{cm}$ spacing between the antennas in either pair corresponding to 0.77 times the wavelength at $2.3 \mathrm{GHz}$. All antennas are omnidirectional.

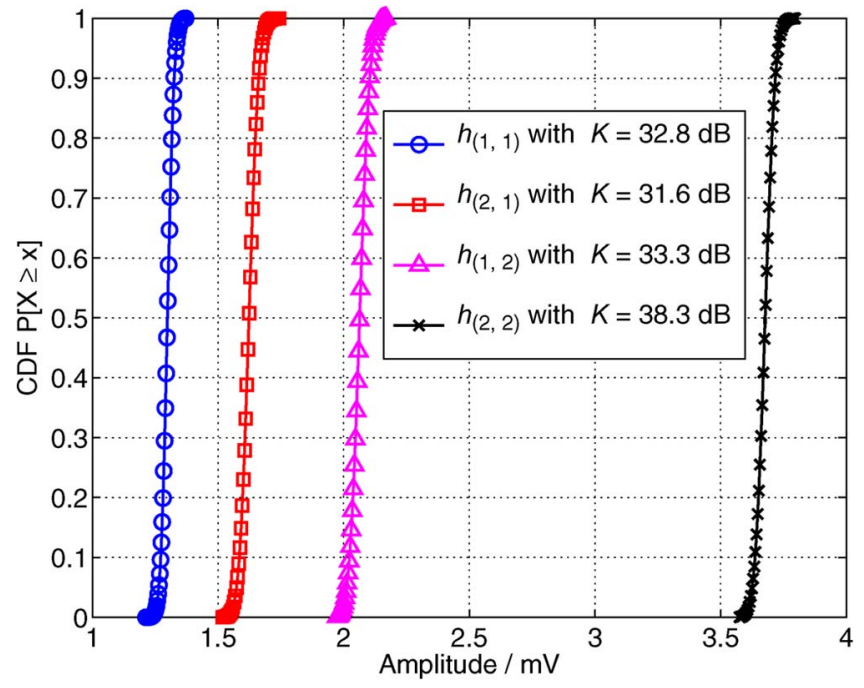

Fig. 9. CDFs for each of the fast-fading coefficients $h_{\left(r, n_{t}\right)}$ of the four channels in the experiment. Each is defined by a Rician distribution with a unique $K$-factor. (Markers) Measurement points. (Lines) Best-fit approximation. Note that the wireless channel mean values fall in the range of $1.3-3.6 \mathrm{mV}$.

where $\mathbf{H}_{r}$ is the received pilot sequence. To enable a more accurate evaluation of the system, the channel is estimated and averaged over ten pilot sequences. Furthermore, two channels are estimated per frame; the first channel estimate is used for the first half of the data symbols in the frame, and the second is used for the second half of the data symbols in the frame.

2.7 Demodulation: The ML optimum receiver for MIMO systems is used, which can be written as

$$
\hat{\mathbf{x}}_{t}^{(\mathrm{ML})}=\underset{\mathbf{x} \in \mathcal{Q}}{\arg \min }\left\{\|\mathbf{y}-\mathbf{H x}\|_{F}^{2}\right\}
$$

where $\mathcal{Q}$ contains every possible $\left(N_{t} \times 1\right)$ transmit vector, and $\hat{\cdot}$ denotes the estimated transmission vector. However, since only one transmit antenna is active at a time for a SM system, the optimal receiver in (13) can be simplified to

$$
\left[\hat{n}_{t}^{(\mathrm{ML})}, \hat{s}_{t}^{(\mathrm{ML})}\right]=\underset{\substack{n_{t} \in\left\{1,2, \ldots, N_{t}\right\} \\ s \in\left\{s_{1}, s_{2}, \ldots, s_{M}\right\}}}{\arg \min }\left\{\sum_{r=1}^{N_{r}}\left|y_{r}-h_{\left(r, n_{t}\right)} s\right|^{2}\right\}
$$

where $y_{r}$ is the $r$ th entries of $\mathbf{y}$.

Finally, the recovered binary data, along with the estimated SNR, are used to obtain the ABER performance of both SM and SMX.

\section{Propagation Environment (Channel)}

The physical layout of the experimental setup is shown in Fig. 7, and the relative antenna spacing is provided in Fig. 8. In particular, the two transmit and two receive antennas are identical and are placed directly across from each other. As such, the channel between the transmitter and the receiver has a strong line-of-sight (LoS) component. Therefore, the channel is assumed to be a Rician fading channel with a large $K$-factor due to the short distance between the transmit and receive antennas, where $K$ is the ratio of the coherent power component, which is usually the LoS, to the noncoherent power components, which is usually the NLoS. The omnidirectional transmit antennas broadcast on a frequency of $2.3 \mathrm{GHz}$ at $10 \mathrm{Ms} / \mathrm{s}$.

Channel measurements were collected to verify that the channel environment followed a Rician distribution. To achieve this, the transmitter broadcasts pulses at $10 \mathrm{Ms} / \mathrm{s}$ on a carrier frequency of $2.3 \mathrm{GHz}$ at $4 \mathrm{dBm}$ peak power. Each pulse includes a FO estimation section, and a total of $10^{5}$ pulse samples are collected. A best-fit approximation is then calculated for the collected data. In particular, a maximum-likelihood estimation is fitted to the collected data. A Chi-squared goodness-of-fit test is then performed to ascertain that the distribution resulting from the maximum-likelihood estimation fits at least $95 \%$ of the data. The empirical cumulative density function (CDF) for each link is shown in Fig. 9. The results show that the channel does follow a Rician distribution with a $K$ factor that ranges between $31-38 \mathrm{~dB}$. The different $K$-factors on the links between the transmit and receive antennas may be explained by the room geometry, the antenna positioning, and the overall propagation environment. However, note that each of the CDFs has a different mean, which will be discussed in the next section.

\section{EQUIPMENT CONSTRAINTS}

Fig. 8 shows the physical layout of the experiment. Note that the $10-\mathrm{cm}$ interantenna separation that is used here is sufficient to guarantee very low, if any, spatial correlation when broadcasting at $2.3 \mathrm{GHz}$ with a 2.2-m separation between the transmitter and the receiver [42].

The physical environment through which the signal passes, starting from the SG-RF at the transmitter until it reaches the 16-Bit Digitizers at the receiver, suffers from connector losses, differences in the RF chains, different phase responses, attenuations, and similar problems. To study and model the 


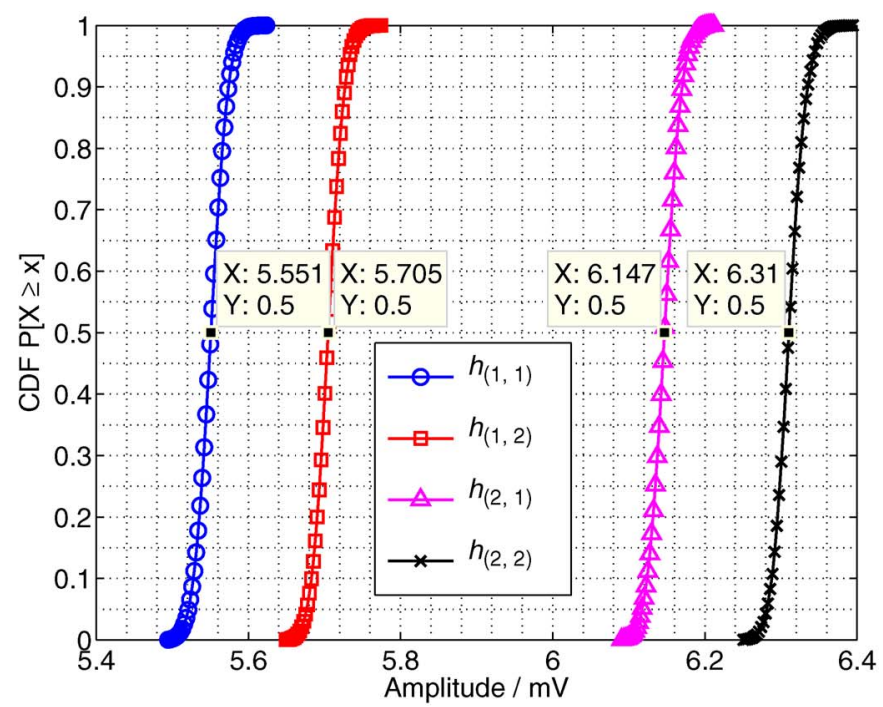

(a)

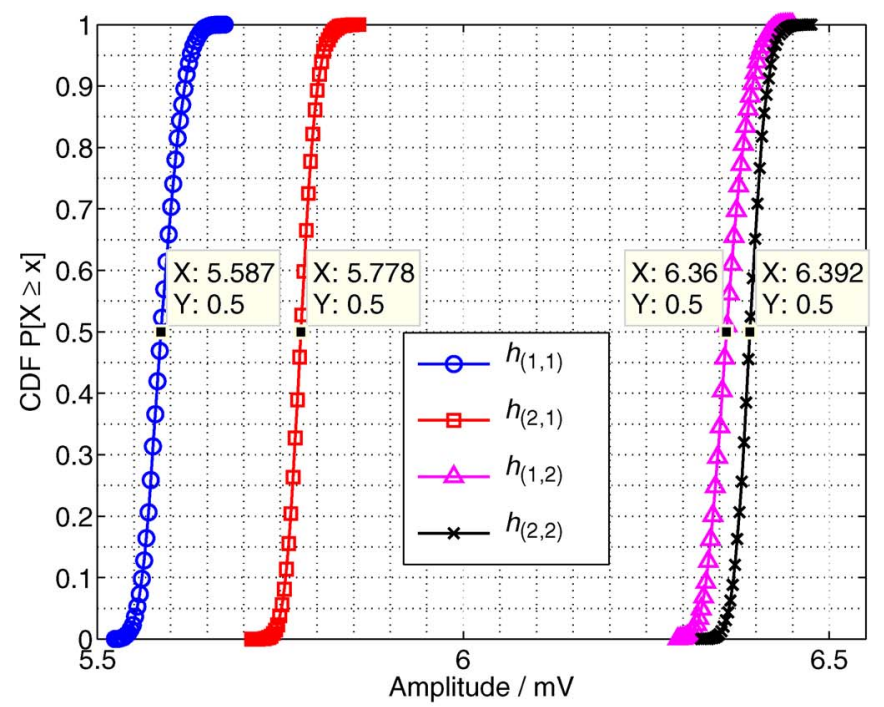

(b)

Fig. 10. CDFs for each of the fast-fading coefficients $h_{\left(r, n_{t}\right)}$ of the four channels in the experiment. Each is defined by a Rician distribution with a unique $K$-factor. (Markers) Measurement points. (Lines) Best-fit approximation. Despite using a coaxial cable with a $10 \mathrm{~dB}$ attenuation to connect the RF chains, each channel exhibits a unique mean. Configurations (a) (I) and (b) (II) of the receive RF chains.

effects of the hardware imperfections on the signal power, we have the following.

1) An RF coaxial cable with a $10 \mathrm{~dB}$ attenuation is connected between each transmit and receive antenna.

2) A pulse is transmitted at $10 \mathrm{Ms} / \mathrm{s}$ on a carrier frequency of $2.3 \mathrm{GHz}$ at $-10 \mathrm{dBm}$ peak power. Each pulse includes an FO estimation section, and a total of $10^{5}$ pulse samples were collected.

3) The CDF for each of the fading coefficients is calculated and is shown in Fig. 10.

In an ideal environment, the means of the CDFs in Fig. 10 should be equal. However, imperfections in the hardware result in different means for each transmit-to-receiver antenna pair, as shown in Fig. 10. The differences between the channels can be modeled as a PI between the various link pairs in the channel matrix $\mathbf{H}$. Therefore, the channel coefficients are redefined as

$$
h_{\left(r, n_{t}\right)}^{\mathrm{PI}}=\sqrt{\alpha_{\left(r, n_{t}\right)}} \times h_{\left(r, n_{t}\right)}
$$

where $\alpha_{\left(r, n_{t}\right)}$ is the channel attenuation coefficient from the receive antenna $r$ to the transmit antenna $n_{t}$.

To locate the source of the discrepancy between the different channel attenuations, i.e., determine if the NI modules or the NI chassis are the source, the RF chains at the receiver were swapped around, and the channels were estimated in configurations (I) and (II). To clarify, configuration (I) represents the default modular setup of the testbed, whereas configuration (II) refers to swapping the front-end modules around the transmit chassis. Fig. 10(a) shows the channel CDF for each transmit-to-receive antenna pair in configuration (I), whereas Fig. 10(b) shows the channel CDF for each transmitto-receive antenna pair in configuration (II). By considering the means of the CDFs in Fig. 10(a) and (b) and taking $h_{(1,1)}$ as a base, the various channel attenuations that result when the receiver is in configuration I or II are given in (16) and (17), shown at the bottom of the page, respectively. Comparing Fig. 10(a) and (b), as well as the attenuations in (16) to those in (17), shows that they are very similar. Indeed, the swapping of the RF chains has a minimal impact on the estimated mean of each channel attenuation. Thus, it can be assumed that the NI modules that compose the receive RF chains are the source of the hardware imperfections and consequently lead to the differences in the means of the estimated CDFs. To account for the hardware imperfections, the channel attenuation coefficients in (16) and (17) are taken into consideration in the derivation of the analytical model in Section IV. The accuracy of the derived analytical bound using the channel attenuation coefficients in the following is demonstrated in Section VI, where it is compared with empirical results.

\section{AnAlytical Modeling}

An analytical model for the ABER performance of the experimental system is developed by considering the system model that is presented in Section II and the system constraints in Section III. The performance of SM and SMX over a single link in a noise-limited scenario is characterized by

$$
\mathrm{ABER} \leq \frac{1}{2^{m}} \sum_{\mathbf{x}_{t}} \sum_{\mathbf{x}} \frac{N\left(\mathbf{x}_{t}, \mathbf{x}\right)}{m} E_{\mathbf{H}}\{\underset{\text { error }}{\operatorname{Pr}}\}
$$

$$
\begin{array}{lll}
\alpha_{(1,1)}=0 \mathrm{~dB}, & \alpha_{(2,1)}=0.25 \mathrm{~dB}, & \alpha_{(1,2)}=0.88 \mathrm{~dB}, \\
\alpha_{(1,1)}=0 \mathrm{~dB}, & \alpha_{(2,2)}=1.1 \mathrm{~dB} \\
\alpha_{(2,1)}=0.29 \mathrm{~dB}, & \alpha_{(1,2)}=1.13 \mathrm{~dB}, & \alpha_{(2,2)}=1.17 \mathrm{~dB}
\end{array}
$$




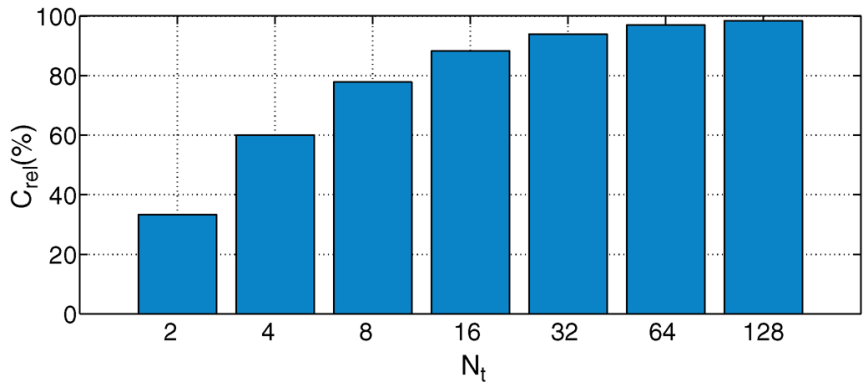

Fig. 11. Relative receiver complexity reduction of the SM-ML receiver versus the SMX-ML receiver.

where $N\left(\mathbf{x}_{t}, \mathbf{x}\right)$ is the number of bits in error between the transmitted vector $\mathbf{x}_{t}$ and $\mathbf{x}, E_{\mathbf{H}}\{\cdot\}$ is the expectation across channel $\mathbf{H}$, and $\operatorname{Pr}_{\text {error }}$ is the conditional pairwise error probability of deciding on $\mathbf{x}$ given that $\mathbf{x}_{t}$ is transmitted [43], i.e.,

$$
\begin{aligned}
\underset{\text { error }}{\operatorname{Pr}} & =\operatorname{Pr}\left(\left\|\mathbf{y}-\mathbf{H} \mathbf{x}_{t}\right\|_{F}^{2}>\|\mathbf{y}-\mathbf{H} \mathbf{x}\|_{F}^{2} \mid \mathbf{H}\right) \\
& =Q\left(\sqrt{\gamma_{\mathrm{ex}}\left\|\mathbf{H}\left(\mathbf{x}_{t}-\mathbf{x}\right)\right\|_{F}^{2}}\right)
\end{aligned}
$$

where $\gamma_{\mathrm{ex}}=\left(E_{m} / 2 N_{0}\right)$ is half of the SNR between the transmitter and the receiver, and $Q(\omega)=(1 / \sqrt{2 \pi}) \int_{\omega}^{\infty} \exp \left(-\left(t^{2} / 2\right)\right) \mathrm{dt}$ is the $Q$-function. As shown in Fig. 8, the transmit and receive antennas in the experiment experience a very strong LoS environment. Accordingly, the channel between each transmitto-receive antenna pair is characterized by Rician fading. A generic Rician channel is defined as

$$
h_{\left(r, n_{t}\right)}=\sqrt{\frac{K}{1+K}}+\sqrt{\frac{1}{1+K}} \widetilde{h}_{\left(r, n_{t}\right)}
$$

where $\widetilde{h}_{\left(r, n_{t}\right)} \sim \mathcal{C N}(0,1)$ is a complex normal circular symmetric random variable with zero mean and unit variance. $n_{t} \in$ $\{1,2\}$ is the index of the transmit antenna, and $r \in\{1,2\}$ is the index of the receive antenna.

To account for the hardware imperfections that result from the PIs, the fast-fading-channel coefficients are redefined according to (15), (16), and (20). Section VI validates the derived analytical bound by comparing it to experimental and simulation results.

\section{Complexity ANAlysis}

The computational complexity of SM-ML is compared with that of the ML detector for SMX (SMX-ML). The complexity is computed as the number of real multiplicative operations $(\times, \div)$ needed by each algorithm. The detailed derivation of each expression is considered in [13] and references therein.

- SMX-ML: The computational complexity of the SMXML receiver that is outlined in (13) is equal to

$$
\mathcal{C}_{\mathrm{SMX}-\mathrm{ML}}=4\left(N_{t}+1\right) N_{r} 2^{m}
$$

where $m$ is the spectral efficiency of the system. Note that $\left(|\mathbf{y}-\mathbf{H x}|^{2}\right)$ in (13) requires $\left(N_{t}+1\right)$ complex multiplications.
- SM-ML: The computational complexity of the SM-ML receiver that is outlined in (14) is equal to

$$
\mathcal{C}_{\mathrm{SM}-\mathrm{ML}}=8 N_{r} 2^{m}
$$

where the ML detector searches through the entire transmit and receive search spaces. Note that evaluating the Euclidean distance $\left(\left|y_{r}-h_{\left(r, n_{t}\right)} s_{t}\right|^{2}\right)$ requires two complex multiplications, where each complex multiplication requires four real multiplications.

Considering (21) and (22), for the same spectral efficiency, the reduction in complexity of the SM-ML receiver relative to that of the SMX-ML receiver is given by

$$
\mathcal{C}_{\text {rel }}=100 \times\left(1-\frac{2}{N_{t}+1}\right) .
$$

On one hand, as shown in (22), the complexity of the SM receiver does not depend on the number of transmit antennas, and it is equal to the complexity of SIMO systems. On the other hand, the complexity of SMX linearly increases with the number of transmit antennas. Therefore, as the number of transmit antennas increases, the relative gain of the SM receiver increases. This is shown in Fig. 11, where the relative complexity for $N_{t} \in\{2,4,8, \ldots, 128\}$ is shown for both systems. In fact, Fig. 11 shows that, for $N_{t}=4$, SM offers a $60 \%$ reduction in complexity, whereas a $98 \%$ reduction in complexity can be seen for $N_{t}=128$. The theoretical, simulation, and empirical results for SM and SMX are now discussed.

\section{EXPERIMENTAL RESUlts AND NUMERICAL ANALYSis}

\section{A. Measurement Campaign}

A stream of $10^{5}$ information bits is sent per transmission to obtain the experimental results. Two transmit antennas are available and binary phase-shift keying is used for the signal constellation. As mentioned in Section II-A2, the real flat bandwidth is $4 \mathrm{MHz}$. The information data are put in 50 frames with 2000 bits each, as shown in the "data section" in Fig. 5. The channel is estimated at the beginning and the end of every frame, resulting in 100 channel estimations per transmission vector. The experiment is repeated 1000 times for every SNR point. In addition, analytical and simulation ABER curves are shown for SM in a Rician environment with and without the PIs that are given in (16).

\section{B. Results}

The simulation, analytical, and experimental results for the ABER performance of SM in an LoS channel are shown in Fig. 12. In particular, the experimental results approximate the performance of the simulation results with PIs, and both the simulation and experimental results are closely approximated by the derived upper bound at a low ABER.

This result serves to validate the theoretical work that is done in the field where the presented SNR along the $x$-axis is equivalent to the SNR on $h_{(1,1)}$. The large error between the experimental, simulation, and analytical curves at high ABER can be attributed to a number of factors, including incorrect FO estimation, timing recovery errors, synchronization problems, 
and poor channel estimation and decoding. Notably, incorrect FO estimation can result in a systematic error that is significantly contributing to the $30 \%$ error that are shown at low SNRs in the figure. As the SNR increases, however, FO estimation, timing recovery, and channel estimation improve, leading to a lower ABER, as shown in Fig. 12. Differences between the measured and simulated ABER curves can be attributed to channel imperfections such as channel correlations, mutual coupling, and interference signals from the surrounding environment. Quantifying these imperfections is deemed important and requires channel modeling and interference measurement. However, addressing these effects is beyond the scope of this paper and will be the subject of future works.

SM performs best in a rich scattering environment where the channel between each transmit and receive antenna is unique. In particular, the larger the Euclidean distance between two received vectors is, the better the ABER performance of SM becomes. Conversely, the more similar the channels are, the worse the ABER of SM is. However, the channel uniqueness can be the result of the scattering environment or PIs that are caused by hardware tolerances. The analytical and simulation results in Fig. 12 show the poor performance of SM in a Rician environment with no PI between the various transmitter-toreceiver links. Fig. 12 also shows the analytical and simulation ABERs for SM when PI are introduced. Indeed, the ABER of SM significantly improves when these PIs are introduced, as each channel becomes more separable. This increases the Euclidean distance and improves performance.

If the channels between each transmit antenna to each receive antenna are similar, then the ABER performance of SM degrades. This is seen when looking at the SM system without PIs, which is shown by the dashed green line with triangular markers in Fig. 12. In fact, the ABER of SM can be approximated by separating the error that originates from the estimation of the spatial-constellation symbol and the error that originates from the estimation of the signal-constellation symbol [44]. Therefore, depending on the environment, the main contributor to the overall ABER of a SM system will be the erroneous detection of the spatial or signal constellation.

When PIs are introduced, the Euclidean distance between the channel signatures increases. This decreases the error contribution of the spatial component of SM. Hence, when the SNR is sufficiently high to have near-perfect channel estimation, the error of the system is bounded by the error from the signal component of SM. This separation can be only shown when iterative detection is used, which is proven to be suboptimal [12]. In addition, in [45], it is shown that the error, when only the spatial constellation of SM is used for data transmission, gets worse for an increasing $K$ factor in a Rician environment. This is the opposite of conventional modulation techniques since a larger $K$ factor for SM means a smaller Euclidean distance between the spatial-constellation points, which results in an increased ABER performance. Indeed, it is the Euclidean distance between the different channels that determines the error in the spatial-constellation detection. However, since MLoptimal detection is used at the receiver, separating the error from the spatial and signal symbols is strictly not permitted. Please note that the PIs between the links are always obtained

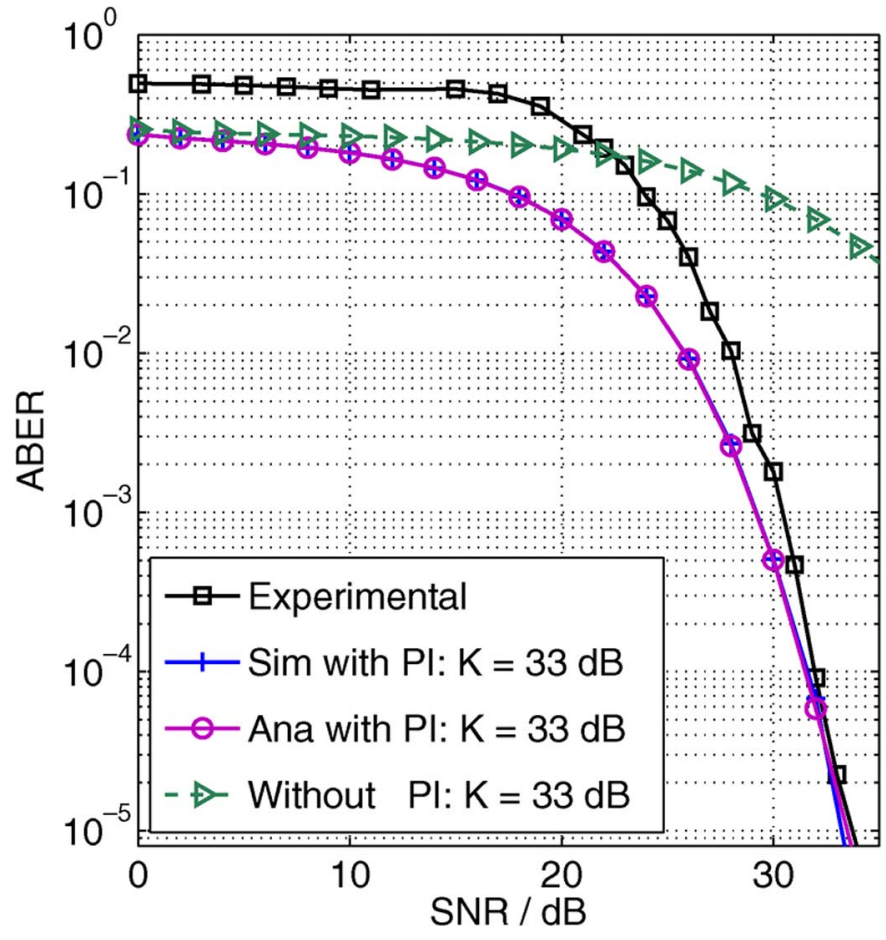

Fig. 12. ABER for SM in an experimental setup with two transmit antennas, two receive antennas, and a spectral efficiency of $2 \mathrm{bits} /\left(\mathrm{s} \cdot \mathrm{Hz}^{-1}\right)$. The SNR is set as measured on $h_{(1,1)}$ with $\alpha_{(1,1)}=0 \mathrm{~dB}$. (Solid black line with square markers) Experimental results. (Green diamond markers) Simulation results with no PI between the links. (Green dashed line) Analytical prediction. The remaining curves denote the simulation (Sim) and analytical (Ana) results.

relative to the channel with the greatest attenuation, i.e., the values of the PI factors in (16) and (17) are always positive.

Furthermore, PIs between the transmitting antennas are shown to offer improved performance in terms of the ABER when only the spatial constellation of SM is used, i.e., when space shift keying (SSK) is the underlying modulation technique. In particular, an optimized power allocation for a various number of transmit antennas is addressed in [23], where the authors show that there is optimal power allocation between the transmitting antennas, which can serve to increases the Euclidean distance between the channel signatures and improve the ABER performance of SM. Indeed, SM has been also successfully applied to an AWGN optical wireless channel, where it is shown that PIs greatly improve the ABER performance [46].

The simulation, analytical, and experimental results for the ABER performance of SMX in a LoS channel are shown in Fig. 13. In particular, the experimental results closely follow the performance of the simulation results with PIs, and both the simulation and experimental results are closely approximated by the derived upper bound at low ABER when the hardware imperfections are taken into account. This result serves to validate the theoretical work that is done in the field. The results in Fig. 13 show that the SMX system, like the SM system, also benefits from the PIs in the hardware. The SMX system approximately exhibits a $3 \mathrm{~dB}$ coding gain when compared with $\mathrm{SM}$ at an ABER of $10^{-4}$. This coding gain is also shown at an ABER of $10^{-3}$ in Fig. 14, where the simulation and analytical results for the ABER performance of SM and SMX are shown when there are no PIs between the links. 


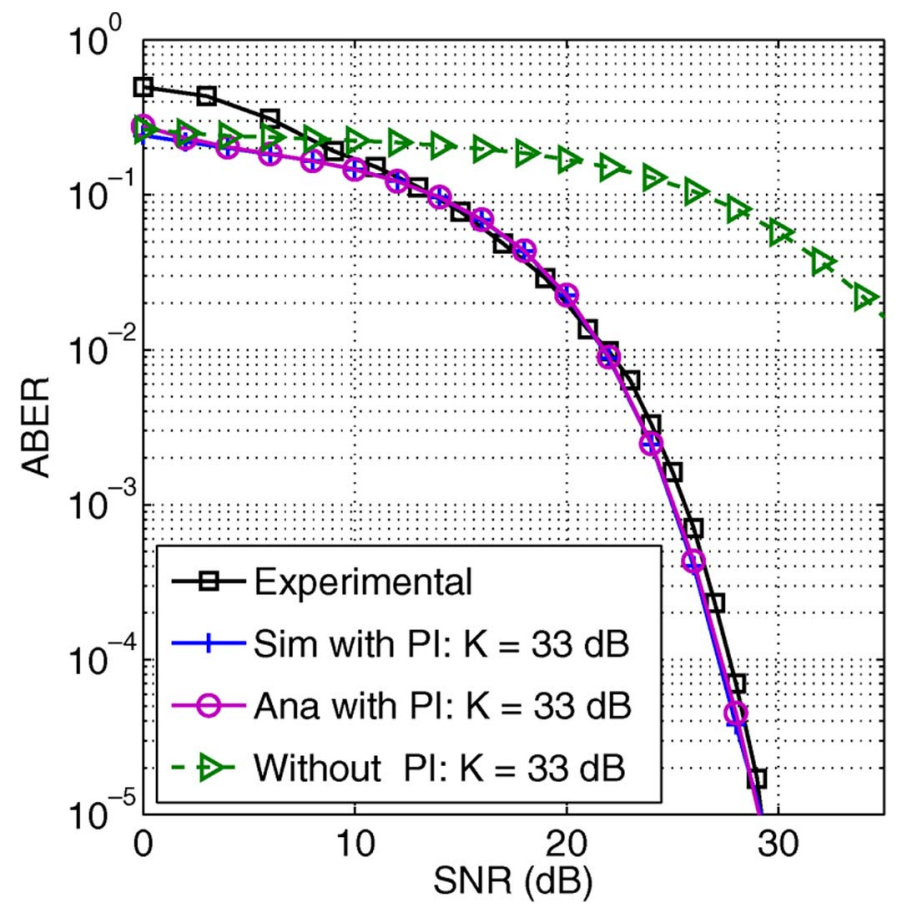

Fig. 13. ABER for SMX in an experimental setup with two transmit antennas, two receive antennas, and a spectral efficiency of $2 \mathrm{bits} /\left(\mathrm{s} \cdot \mathrm{Hz}^{-1}\right)$. The SNR is set as measured on $h_{(1,1)}$ with $\alpha_{(1,1)}=0 \mathrm{~dB}$. (Solid black line with square markers) Experimental results. (Green diamond markers) Simulation results with no PI between the links. (Green dashed line) Analytical prediction. The remaining curves denote the simulation (Sim) and analytical (Ana) results.

The coding gain of SMX relative to SM is expected when there are few transmit antennas. The Euclidean distance between the transmit vectors and, therefore, the variance in (19) in SMX is larger than in SM. However, the aim of this paper is to show that empirical results validate the simulation and analytical works that are done in the field, which are shown in both Figs. 12 and 13. Unfortunately, due to the limited number of transmitter and receiver RF chains that are available, there are no experimental results for systems with a larger number of transmit or receive antennas where SM is shown to perform better than SMX. These empirical results will be the focus of future research. Nonetheless, the accuracy of the theoretical and simulation results of SMX and SM with a large number of transmit and receive antennas can be extrapolated from the presented results.

Fig. 15 compares the ABER between SM (see solid lines) and SMX (see dashed lines) in a system with a large number of transmit antennas. Each system operates in a Rayleigh fading environment with a spectral efficiency of $8 \mathrm{bits} /\left(\mathrm{s} \cdot \mathrm{Hz}^{-1}\right)$ and four receive antennas. The results demonstrate the coding gains available to a SM system, as compared with SMX when a large number of transmit antennas are available. In particular, SM with $N_{t}=64$ offers a coding gain of up to $4 \mathrm{~dB}$ with respect to SMX with $N_{t}=8$ and a coding gain of $6 \mathrm{~dB}$ with respect to SMX with $N_{t}=4$. These performance gains stem from the greater Euclidean distance between the transmit vectors for SM. It is important to note that, although SM is simulated as having 64 transmit antennas available, it requires only a single RF chain, whereas SMX requires eight RF chains for the eight transmit antennas. Furthermore, to achieve the

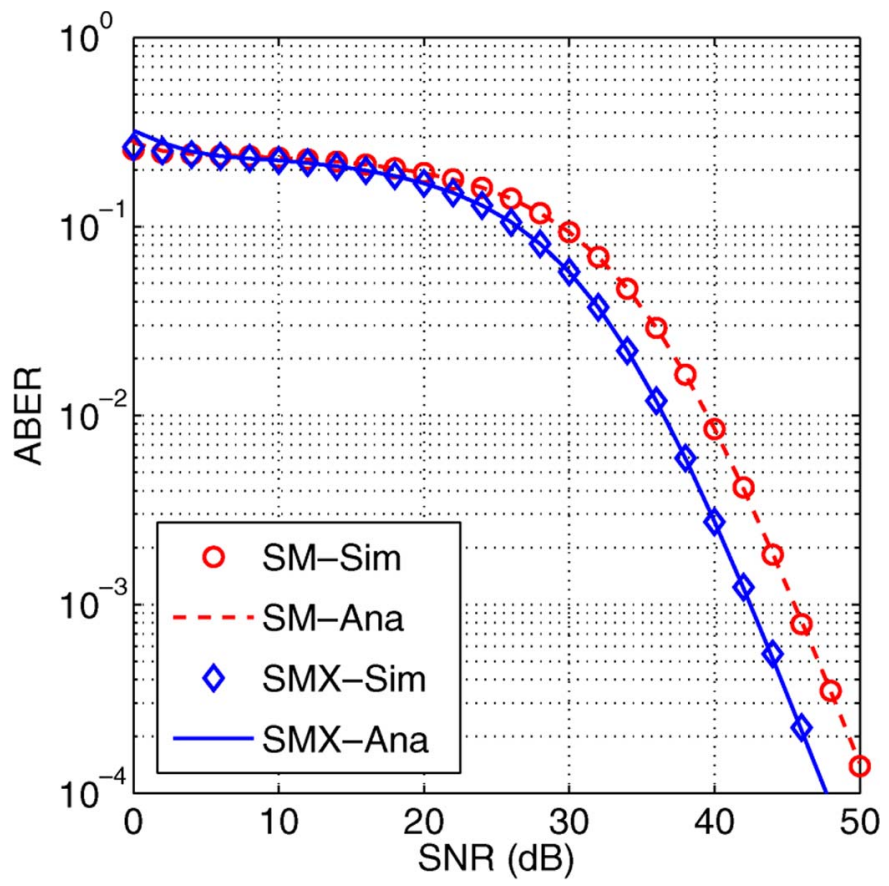

Fig. 14. ABER for SM and SMX in a Rician fading channel where $K=$ $33 \mathrm{~dB}$ with two transmit antennas, two receive antennas, a spectral efficiency of $2 \mathrm{bits} /\left(\mathrm{s} \cdot \mathrm{Hz}^{-1}\right)$, and no PIs between the channels. (Markers) Simulation (Sim) and (lines) analytical (Ana) results.

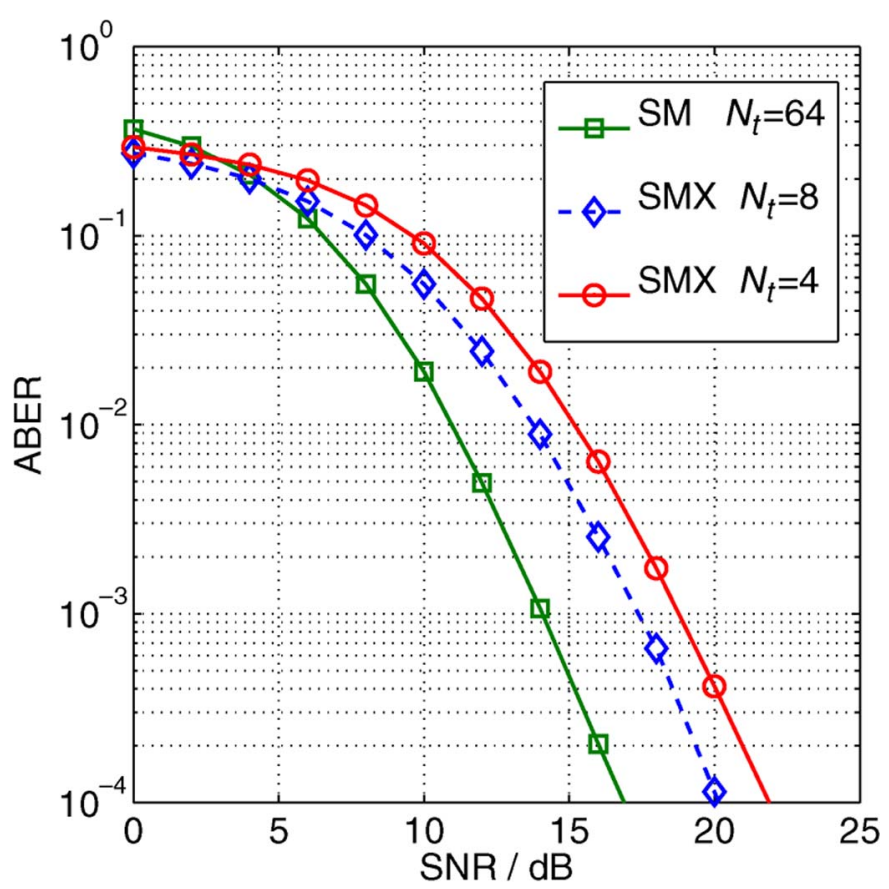

Fig. 15. Simulation results for the ABER for SM and SMX in a Rayleigh fading environment with a spectral efficiency of $8 \mathrm{bits} /\left(\mathrm{s} \cdot \mathrm{Hz}^{-1}\right)$ and no PIs between the channels.

ABER performance that is shown in Fig. 15, SM requires 64 unique channels. In this paper, a unique channel is assumed to be available only with the addition of a single transmit antenna. However, the work in [6], [25], and others, look at creating multiple channel signatures without the need for a large number of physical transmit antennas while maintaining a similar ABER performance to the traditional SM scheme. 
This paper demonstrates that the hardware tolerances of practical communication systems are beneficial for the ABER performance of both SM and SMX. This behavior, along with the requirement for a single RF chain, makes SM a viable candidate for future wireless networks.

\section{Summary AND CONCLUSION}

In this paper, the ABER performance of SM and SMX has been experimentally validated for the first time. In particular, the encoding and decoding processes have been presented. The experimental testbed, equipment, and channel conditions have been described in detail, and the ABERs of SM and SMX have been obtained in a practical testbed environment. In addition, the experimental results have been compared with both simulation and analytical approaches. As a result, it has been shown that a Rician channel with different channel attenuations closely describe the behavior of SM and SMX in the physical environment. Furthermore, it has been demonstrated that the different channel attenuations resulted from various hardware imperfections at the transmitter and receiver RF chains. In fact, the induced PIs resulted in significant coding gains for the practical systems that are relative to the theoretical predictions without such PIs. To this extent, SM and SMX performed as expected, relative to the theoretical work when the PIs have been introduced in the analytical model. This result validated the SM principle. The performance gains exhibited by SM in the practical implementation make SM a viable candidate for future wireless networks and, particularly, for systems with a large number of transmit antennas available.

It is worth noting that this paper may be extended in a number of different ways that would broaden its applicability. Empirical results that demonstrate the performance of SM and SMX with a large number of transmit and receive antennas remain to be obtained. In light of the aforementioned results, the ABER performance of SM and SMX is expected to follow the theoretical models, but these results are essential to validate the ABER performance for both SM and SMX systems. In addition, channel imperfections such as channel correlations and mutual antenna coupling, along with the impact of interfering signals from neighboring transmitters on the same frequency, should be analyzed. Furthermore, obtaining empirical results for the capacity and the energy efficiency of SM are of great interest for future research, particularly since SM is projected to have large energy efficiency gains when compared with other traditional MIMO schemes since it requires only a single RF chain. As a consequence, the quiescent power and circuit power can be kept at low levels. Acquiring the hardware, which would enable the accurate measurement of these aspects, is key. Finally, the implementation of the SM detection algorithm on a DSP or a fieldprogrammable gate array (FPGA) brings with it a number of optimization challenges such as the use of multithreading, pipelining, fixed-point computations, and others. The deployment of SM on an FPGA or a DSP has yet to be demonstrated.

It has been shown that SM is a simple low-cost MIMO technique, which has now demonstrated excellent performance in an LoS wireless channel. Therefore, this paper has shown that $\mathrm{SM}$ is a promising practical approach to obtaining the enhanced performance of SMX without introducing high processor complexity and high power consumption that would occur when using other SMX approaches. The aim now is to investigate the performance of SM in a range of experimental channel conditions and further study its potential.

\section{REFERENCES}

[1] E. Telatar, "Capacity of multi-antenna Gaussian channels," Eur. Trans. Telecommun., vol. 10, no. 6, pp. 585-595, Nov./Dec. 1999.

[2] J. Mietzner, R. Schober, L. Lampe, W. H. Gerstacker, and P. A. Höeher, "Multiple-antenna techniques for wireless communications-A comprehensive literature survey," IEEE Commun. Surveys Tuts., vol. 11, no. 2, pp. 87-105, Second Quart., 2009.

[3] R. Mesleh, H. Haas, Y. Lee, and S. Yun, "Interchannel interference avoidance in MIMO transmission by exploiting spatial information," in Proc. 16th IEEE Int. Symp. PIMRC, Berlin, Germany, 2005, vol. 1, pp. 141-145.

[4] R. Mesleh, H. Haas, S. Sinanovi, C. W. Ahn, and S. Yun, "Spatial modulation," IEEE Trans. Veh. Technol., vol. 57, no. 4, pp. 2228-2241, Jul. 2008.

[5] N. Serafimovski, M. Di Renzo, S. Sinanovi, R. Y. Mesleh, and H. Haas, "Fractional bit encoded spatial modulation (FBE-SM)," IEEE Commun. Lett., vol. 14, no. 5, pp. 429-431, May 2010.

[6] A. Younis, N. Serafimovski, R. Mesleh, and H. Haas, "Generalised spatial modulation," in Proc. Asilomar Conf. Signals, Syst. Comput., Pacific Grove, CA, USA, Nov. 2010, pp. 1498-1502.

[7] J. Jeganathan, A. Ghrayeb, L. Szczecinski, and A. Ceron, "Space shift keying modulation for MIMO channels," IEEE Trans. Wireless Commun., vol. 8, no. 7, pp. 3692-3703, Jul. 2009.

[8] G. Auer, V. Giannini, C. Desset, I. Godor, P. Skillermark, M. Olsson, M. Imran, D. Sabella, M. Gonzalez, O. Blume, and A. Fehske, "How much energy is needed to run a wireless network?" IEEE Wireless Commun., vol. 18, no. 5, pp. 40-49, Oct. 2011.

[9] C. Desset, B. Debaillie, V. Giannini, A. Fehske, G. Auer, H. Holtkamp, W. Wajda, D. Sabella, F. Richter, M. J. Gonzalez, H. Klessig, I. Godor, M. Olsson, M. A. Imran, A. Ambrosy, and O. Blume, "Flexible power modeling of LTE base stations," in Proc. IEEE WCNC, Shanghai, China, Apr. 1-4, 2012, pp. 2858-2862.

[10] A. Stavridis, S. Sinanovi, M. D. Renzo, H. Haas, and P. Grant, "Energy saving base station employing spatial modulation," Proc. IEEE 17th Int. Workshop CAMAD, pp. 231-235, Sep. 17-19, 2012.

[11] A. Stavridis, S. Sinanovi, M. D. Renzo, and H. Haas, "A power saving dual-hop architecture based on hybrid spatial modulation," in Conf. Rec. 46th ASILOMAR, Nov. 4-7, 2012, pp. 1366-1370.

[12] J. Jeganathan, A. Ghrayeb, and L. Szczecinski, "Spatial modulation: Optimal detection and performance analysis," IEEE Commun. Lett., vol. 12, no. 8, pp. 545-547, Aug. 2008.

[13] A. Younis, S. Sinanovic, M. Di. Renzo, R. Mesleh, and H. Haas, "Generalised sphere decoding for spatial modulation," IEEE Trans. Commun., vol. 61, no. 7, pp. 2805-2815, Jul. 2013.

[14] A. Younis, R. Mesleh, H. Haas, and P. M. Grant, "Reduced complexity sphere decoder for spatial modulation detection receivers," in Proc. IEEE GLOBECOM, Miami, FL, USA, Dec. 2010, pp. 1-5.

[15] A. Younis, M. Di Renzo, R. Mesleh, and H. Haas, "Sphere decoding for spatial modulation," in Proc. IEEE ICC, Kyoto, Japan, 2011, pp. 1-6.

[16] R. Mesleh, M. Di Renzo, H. Haas, and P. M. Grant, "Trellis coded spatial modulation," IEEE Trans. Wireless Commun., vol. 9, no. 7, pp. 23492361, Jul. 2010.

[17] M. Di Renzo and H. Haas, "A general framework for performance analysis of space shift keying (SSK) modulation for MISO correlated Nakagami-m fading channels," IEEE Trans. Commun., vol. 58, no. 9, pp. 2590-2603, Sep. 2010.

[18] S. U. Hwang, S. Jeon, S. Lee, and J. Seo, "Soft-output ML detector for spatial modulation OFDM systems," IEICE Electron. Exp., vol. 6, no. 19, pp. 1426-1431, Oct. 2009.

[19] M. Di Renzo and H. Haas, "Space shift keying (SSK) modulation with partial channel state information: Optimal detector and performance analysis over fading channels," IEEE Trans. Commun., vol. 58, no. 11, pp. 3196-3210, Nov. 2010.

[20] S. S. Ikki and R. Mesleh, "A general framework for performance analysis of space shift keying (SSK) modulation in the presence of Gaussian imperfect estimations," IEEE Commun. Lett., vol. 16, no. 2, pp. 228-230, Feb. 2012. 
[21] E. Basar, U. Aygolu, E. Panayirci, and H. V. Poor, "Performance of spatial modulation in the presence of channel estimation errors," IEEE Commun. Lett., vol. 16, no. 2, pp. 176-179, Feb. 2012.

[22] M. D. Renzo, D. D. Leonardis, F. Graziosi, and H. Haas, "Space shift keying (SSK) MIMO with practical channel estimates," IEEE Trans. Commun., vol. 60, no. 4, pp. 998-1012, Apr. 2012.

[23] M. Di Renzo and H. Haas, "Improving the performance of space shift keying (SSK) modulation via opportunistic power allocation," IEEE Commun. Lett., vol. 14, no. 6, pp. 500-502, Jun. 2010.

[24] T. Handte, A. Muller, and J. Speidel, "BER analysis and optimization of generalized spatial modulation in correlated fading channels," in Proc. IEEE VTC Fall, Anchorage, AK, USA, Sep. 20-23, 2009, pp. 1-5.

[25] E. Basar, U. Aygolu, E. Panayirci, and V. H. Poor, "Space-time block coded spatial modulation," IEEE Trans. Commun., vol. 59, no. 3, pp. 823832, Mar. 2011.

[26] M. Di Renzo and H. Haas, "On transmit-diversity for spatial modulation MIMO: Impact of spatial-constellation diagram and shaping filters at the transmitter," IEEE Trans. Veh. Technol., vol. 62, no. 6, pp. 2507-2531, Jul. 2013.

[27] N. Serafimovski, S. Sinanovic, M. Di Renzo, and H. Haas, "Dual-hop spatial modulation (Dh-SM)," in Proc. IEEE VTC Spring, Budapest, Hungary, May 15-18, 2011, pp. 1-5.

[28] A. Stavridis, S. Sinanovi, M. D. Renzo, and H. Haas, "Energy evaluation of spatial modulation at a multi-antenna base station," in Proc. 78th IEEE VTC, Las Vegas, NV, USA, Sep. 2-5, 2013.

[29] M. Di Renzo and H. Haas, "Bit error probability of spatial modulation (SM) MIMO over generalized fading channels," IEEE Trans. Veh. Technol., vol. 61, no. 3, pp. 1124-1144, Mar. 2012.

[30] A. Younis, W. Thompson, M. D. Renzo, C.-X. Wang, M. A. Beach, H. Haas, and P. M. Grant, "Performance of spatial modulation using measured real-world channels," in Proc. 78th IEEE VTC, Las Vegas, NV, USA, Sep. 2-5, 2013.

[31] M. Di Renzo, H. Haas, and P. M. Grant, "Spatial modulation for multipleantenna wireless systems: A survey," IEEE Commun. Mag, vol. 49, no. 12, pp. 182-191, Dec. 2011.

[32] "16-Bit IF Digitizer with Onboard Signal Processing," NI PXIe-5622 Specifications, National Instruments, 2011.

[33] P. Chambers, X. Hong, Z. Chen, C.-X. Wang, M. Beach, and H. Haas, "The UC4G wireless MIMO testbed," in Proc. IEEE GLOBECOM, Anaheim, CA, USA, Dec. 3-7, 2012, pp. 4368-4373.

[34] G. J. Foschini, "Layered space-time architecture for wireless communication in a fading environment when using multi-element antennas," Bell Labs Tech. J., vol. 1, no. 2, pp. 41-59, 1996.

[35] S. Tiiro, J. Ylioinas, M. Myllyla, and M. Juntti, "Implementation of the least squares channel estimation algorithm for MIMO-OFDM systems," in Proc. Int. ITG WSA, Berlin, Germany, 2009.

[36] L. Lo Presti and M. Mondin, "Design of optimal FIR raised-cosine filters," Electron. Lett., vol. 25, no. 7, pp. 467-468, Mar. 1989.

[37] J. L. Massey, "Optimum frame synchronization," IEEE Trans. Commun., vol. 20, no. 2, pp. 115-119, Apr. 1972.

[38] H. Xuefei and C. Jie, "Implementation frame synchronization for MIMOOFDM system with ZCZ-codes," in Proc. IEEE Int. Symp. MAPE, 2005, vol. 1, pp. 241-244.

[39] J.-J. van de Beek, M. Sandell, M. Isaksson, and P. Ola Borjesson, "Low-complex frame synchronization in OFDM systems," in Proc. IEEE Int. Conf. Universal Pers. Commun., Tokyo, Japan, Nov. 6-10, 1995, pp. 982-986.

[40] H. Hashemi, "The indoor radio propagation channel," Proc. IEEE, vol. 81, no. 7, pp. 943-968, Jul. 1993.

[41] D. McNamara, M. Beach, and P. Fletcher, "Experimental investigation of the temporal variation of MIMO channels," in Proc. IEEE 54th VTC Fall, Atlantic City, NJ, USA, 2001, vol. 2, pp. 1063-1067.

[42] F. Quitin, C. Oestges, F. Horlin, and P. D. Doncker, "Multipolarized MIMO channel characteristics: analytical study and experimental results," IEEE Trans. Antennas Propag., vol. 57, no. 9, pp. 2739-2745, Sep. 2009.

[43] M. D. Renzo and H. Haas, "Performance analysis of spatial modulation," in Proc. Int. ICST Conf. CHINACOM, Aug. 2010, pp. 1-7.

[44] M. Di Renzo and H. Haas, "Bit error probability of space modulation over Nakagami-m fading: Asymptotic analysis," IEEE Commun. Lett., vol. 15, no. 10 , pp. 1026-1028, Oct. 2011.

[45] M. D. Renzo and H. Haas, "Space shift keying (SSK-) MIMO over correlated Rician fading channels: Performance analysis and a new method for transmit-diversity," IEEE Trans. Commun., vol. 59, no. 1, pp. 116-129, Jan. 2011.

[46] T. Fath, H. Haas, M. Di Renzo, and R. Mesleh, "Spatial modulation applied to optical wireless communications in indoor LOS environments," in Proc. IEEE GLOBECOM, Houston, TX, USA, 2011, pp. 1-5.

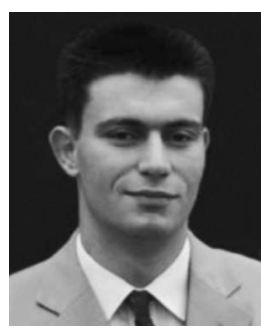

Nikola Serafimovski received the B.Sc. degree in electrical engineering and computer science and the M.Sc. degree in communications, systems, and electronics from Jacobs University, Bremen, Germany, in 2007 and 2009, respectively, and the Ph.D. degree from The University of Edinburgh, Edinburgh, U.K., where the focus of his research was on multiple-input multiple-output systems and the practical implementation of spatial modulation.

$\mathrm{He}$ is currently a Communication Systems Engineer with pureVLC Ltd, Edinburgh, U.K.

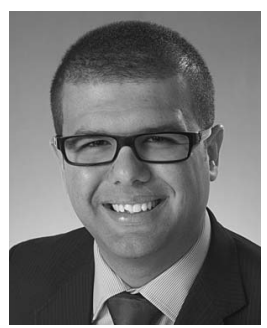

Abdelhamid Younis received the B.Sc. degree in electrical and electronic engineering (with honors) in 2007 from the University of Benghazi, Benghazi, Libya, and the M.Sc. degree in signal processing and communication engineering (with distinction) in 2009 from The University of Edinburgh, Edinburgh, U.K., where he is currently working toward the Ph.D. degree in communication engineering in the Institute of Digital Communications.

His main research interests are in the area of wireless communication and digital signal processing with a particular focus on spatial modulation, multiple-input multiple-output (MIMO) wireless communications, reduced complexity MIMO design, and optical wireless communications.

Mr. Younis received the Overseas Research Student Award in 2010 in recognition of his work.

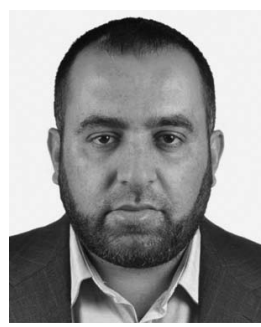

Raed Mesleh (S'00-M'08-SM'13) received the $\mathrm{Ph} . \mathrm{D}$. degree in electrical engineering from Jacobs University, Bremen, Germany.

$\mathrm{He}$ had several years of postdoctoral wirelesscommunication and optical-wireless-communication research experience in Germany. Since October 2010, he has been with the University of Tabuk, Tabuk, Saudi Arabia, where he is currently an Assistant Professor and the Director of the research excellence unit. Since 2007, his publications have received more than 800 citations. He has published more than 50 publications in top-tier journals and conferences. He is the holder of seven granted patents. His main research interests are in spatial modulation, multipleinput multiple-output cooperative wireless communication techniques, and optical wireless communication.

Dr. Mesleh serves on the Technical Program Committee for academic conferences and is a regular reviewer for most of the journals of the IEEE/Optical Society of America (OSA) Communication Society and the IEEE/OSA Photonics Society.

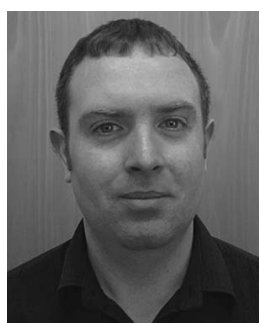

P. Chambers received the B.Sc. degree in physics and physics technology and the Ph.D. degree in transmission line and wireless communications from the Dublin Institute of Technology, Dublin, Ireland, in 2002 and 2008, respectively.

From to 2009 to 2010, he worked as a Postdoctoral Researcher with Université Catholique de Louvain, Louvain-la-Neuve, Belgium. Then, he worked as a Research Associate on the U.K.-China Science Bridges: Research and Development on Beyond Fourth-Generation Wireless Mobile Communications (UC4G) Project. From 2010 to 2013, he was with the Defence Science and Technology Laboratory, Heriot-Watt University, Edinburgh, U.K., working on on the development of cognitive radio algorithms. He is currently a Research Associate with the University of Surrey, Surrey, U.K., where he is working on fifth-generation communications standards as part of the Low-ElectromagneticField Exposure Network (LexNet) Project Team. 


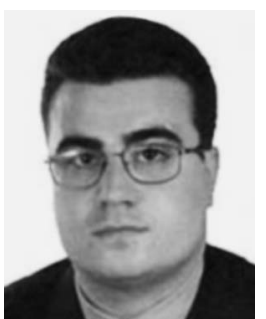

Marco Di Renzo (S'05-AM'07-M'09) was born in L'Aquila, Italy, in 1978. He received the Laurea (cum laude) and Ph.D. degrees in electrical and information engineering from the University of L'Aquila, Coppito, Italy, in 2003 and 2007, respectively.

From August 2002 to January 2008, he was with the Center of Excellence for Research "Design Methodologies of Embedded Controllers, Wireless Interconnect and Systems-on-chip (DEWS)," University of L'Aquila. From February 2008 to April 2009, he was a Research Associate with the Telecommunications Technological Center of Catalonia (CTTC), Barcelona, Spain. From May 2009 to December 2009, he was an Engineering and Physical Sciences Research Council Research Fellow with the Institute for Digital Communications, The University of Edinburgh, Edinburgh, U.K. Since January 2010, he has been a Tenured Researcher ("Chargé de Recherche Titulaire") with the French National Center for Scientific Research (CNRS), as well as a Faculty Member with the Laboratory of Signals and Systems, a joint research laboratory of the CNRS, the École Supérieure d' Électricité, and the University of ParisSud XI, Paris, France. His main research interests are in the area of wireless communications theory. He is a Principal Investigator of three European-funded research projects (Marie Curie ITN-GREENET, Marie Curie IAPP-WSN4QoL, and Marie Curie ITN-CROSSFIRE)

Dr. Di Renzo received the Special Mention for the Outstanding Five-Year (1997-2003) Academic Career, University of L'Aquila; the Thales Communications fellowship for doctoral studies (2003-2006), University of L'Aquila; the Best Spinoff Company Award (2004), Abruzzo Region, Italy; the Torres Quevedo award for research on ultrawideband systems and cooperative localization for wireless networks (2008-2009), Ministry of Science and Innovation, Spain; the "Dérogation pour l'Encadrement de Thèse" (2010), University of Paris-Sud XI, France; the 2012 IEEE International Workshop on ComputerAided Modeling Analysis and Design of Communication Links and Networks Best Paper Award from the IEEE Communications Society; and the 2012 Exemplary Reviewer Award from the IEEE WiRELESS COMMUNICATIONS LETTERS of the IEEE Communications Society. He currently serves as an Editor of the IEEE COMMUNICATIONS LETTERS.

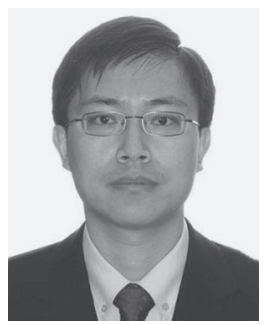

Cheng-Xiang Wang (S'01-M'05-SM'08) received the B.Sc. and M.Eng. degrees in communication and information systems from Shandong University, Jinan, China, in 1997 and 2000, respectively, and the Ph.D. degree in wireless communications from Aalborg University, Aalborg, Denmark, in 2004.

Since 2005, he has been with Heriot-Watt University, Edinburgh, U.K., where he was first a Lecturer, then a Reader in 2009, and has been a Professor since 2011. He is also an Honorary Fellow with The University of Edinburgh, U.K., and a Chair/Guest Professor with Shandong University, Huazhong University of Science and Technology, Wuhan, China, and the Southeast University, Nanjing, China. He was a Research Fellow with the University of Agder, Grimstad, Norway, from 2001 to 2005, a Visiting Researcher with Siemens AG-Mobile Phones, Munich, Germany, in 2004, and a Research Assistant with the Technical University of Hamburg-Harburg, Hamburg, Germany, from 2000 to 2001. He has published one book chapter and over 180 papers in refereed journals and conference proceedings. His current research interests include wireless channel modeling and simulation, green communications, cognitive radio networks, vehicular communication networks, large multiple-input multiple-output (MIMO), cooperative MIMO, and Beyond Fourth-Generation wireless communications.

Prof. Wang served or is currently serving as an editor for eight international journals, including the IEEE TRANSACTIONS ON VEHICULAR TECHNOLOGY (2011 to present) and the IEEE TRANSACTIONS ON WIRELESS COMMUNICATIONS (2007-2009). He was the Guest Editor for the IEEE JOURNAL on Selected Areas in Communications, Special Issue on Vehicular Communications and Networks and Special Issue on Spectrum and Energy Efficient Design of Wireless Communication Networks. He served or is serving as a Technical Program Committee (TPC) member, the TPC Chair, and the General Chair for over 70 international conferences. He received the Best Paper Awards from the IEEE Global Communications Conference in 2010, the IEEE International Conference on Communication Technology in 2011, and The 12th International Conference on ITS Telecommunications (ITST 2012). He is a Fellow of the Institution of Engineering and Technology, a Fellow of the Higher Education Academy, and a member of the Engineering and Physical Sciences Research Council Peer-Review College.

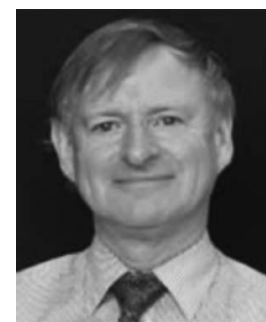

Peter M. Grant (M'77-SM'83-F'96) was born in St. Andrews, U.K. He received the B.Sc. degree in electronic engineering from the Heriot-Watt University, Edinburgh, U.K., in 1966, the Ph.D. degree from The University of Edinburgh in 1975, and honorary D.Eng. degrees from the Heriot-Watt University in 2006 and from Napier University, Edinburgh, in 2007 .

$\mathrm{He}$ worked in radio communications with the Plessey Company. He was then appointed to a research fellowship with The University of Edinburgh, where he was subsequently promoted to a Full Professor of electronic signal processing and a Departmental Chair. From 2002 to 2008, he served as the Head of the School of Engineering and Electronics, Edinburgh. During the academic year 1977-1978, he was a Visiting Professor with the Ginzton Laboratory, Stanford University, Stanford, CA, USA, and from 1985 to 1986, he was a Visiting Staff Member with the Lincoln Laboratory, Massachusetts Institute of Technology, Lexington, MA, USA.

Prof. Grant received the 82nd Faraday Medal from the Institution of Electrical Engineers (IEE) for his work on code-division multiple-access receiver designs and adaptive filters in 2004, the Bulgin premium in 1974 and 1977 and the Lord Mountbatten premium in 1982 from the then Institution of Electronic and Radio Engineers, and both the IEE Marconi and Langham Thompson premia in 1994. From 1980 to 1996, he served as an Honorary Editor of the IEE Proceedings entitled "Vision Image and Signal Processing." He was a member in 1992 and 1996 and the Chair of the 2001 Universities Funding Council research assessment panel for the U.K. Electrical Engineering Departments. He was the Technical Program Chairman for the International Conference on Acoustics, Speech, and Signal Processing in 1989, the Chairman of the European Signal Processing Conference in 1994, and the President of the European Association for Signal Processing (EURASIP) from 2000 to 2002. In 1998, he was appointed by the IEEE Signal Processing Society as a Distinguished Lecturer on digital signal processing for mobile communications. He served as the Director of the Virtual Centre of Excellence In Mobile and Personal Communications Limited from 2007 to 2009. In 2007, he was appointed to be the Eighth Regius Professor of Engineering with The University of Edinburgh. In 2009, he was made an Officer of the Order of the British Empire in the Queen's birthday honors list. He holds fellowships of the Institution of Engineering and Technology, the Royal Academy of Engineering, and the Royal Society of Edinburgh.

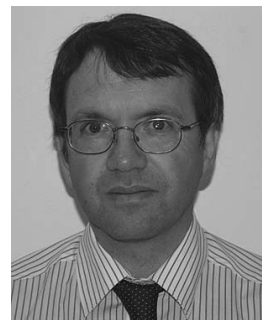

Mark A. Beach received the Ph.D. degree from the University of Bristol, Bristol, U.K., in 1987.

He was a Research Assistant from September 1987 to July 1989, a Lecturer from August 1989 to July 1996, a Senior Lecturer from August 1996 to July 1998, a Reader in communication systems from August 1998 to July 2003, the Head of the Department of Electrical and Electronic Engineering from August 2006 to July 2010, and has been a Professor of radio systems engineering since August 2003 with the University of Bristol.

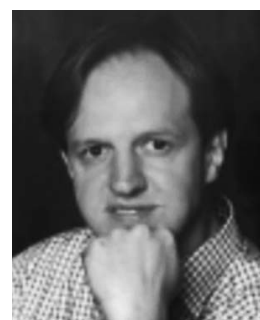

Harald Haas (S'98-A'00-M'03) received the $\mathrm{Ph} . \mathrm{D}$. degree from The University of Edinburgh, Edinburgh, U.K., in 2001

$\mathrm{He}$ is the Chair of Mobile Communications with the Institute for Digital Communications, The University of Edinburgh, and he is currently the chief technical officer of a university spinout company pureVLC Ltd. He is the holder of 23 patents. He has published more than 60 journal papers, including a Science article and more than 160 peer-reviewed conference papers. Nine of his papers are invited papers. He has coauthored a book entitled Next Generation Mobile Access Technologies: Implementing TDD (Cambridge University Press). Since 2007, he has been a Regular High-Level Visiting Scientist supported by the Chinese 111-program with Beijing University of Posts and Telecommunications, Beijing, China. He was an invited speaker at the Technology, Entertainment, Design Global Conference in 2011. His main research interests are in interference coordination in wireless networks, spatial modulation, and optical wireless communication.

Prof. Haas received a prestigious Fellowship of the Engineering and Physical Sciences Research Council, U.K. His work on optical wireless communication was listed among the "50 Best Inventions in 2011" in Time Magazine. 